In the manuscript of the paper we initially included more information, but we were advised that the reader could be overwhelmed by the mass of data and that it would be better to highlight significant associations. From the point of view of most readers this is clearly good advice, although for those who are particularly interested in the data set it may be less than satisfactory. We would be pleased to provide interested colleagues with details of any particular aspect if they write to us directly.

We regret that we have expressed ourselves less than clearly with regard to the classification of patients' occupational outcome. Patients who were unemployed at follow-up were classed as unemployed even if they had also been unemployed at the time of the index admission. As may be seen from Table 5 of the paper, we did initially attempt a more refined classification of employment status, but the numbers who were employed in a higher status job were small and the numbers still in work but in a lower status not much greater. It is on the data in Table 5 that the result whereby good occupational outcome in patients with a relatively short pre-treatment duration was associated with the prescription of placebo medication during the follow-up period depends. All seven patients whose occupational status improved had a relatively short pre-treatment duration of illness. Six of them were prescribed placebo medication.

Dr Muijen indicates that he thinks we should not class patients as having a poor outcome because they are unemployed. There are many ways of looking at outcome in patients with illnesses which may be associated with long-term disability. For example, outcome in these patients has been considered in terms of relapse, death, in-patient days, judicial contact, ability to give child care and the rather non-specific concept of achievement. None of these measures, including employment status, is ideal and we understand from discussions with colleagues at the Maudsley Hospital that patients from their catchment areas are frequently unemployed and, even if they are reasonably well, are not likely to find work. This is not true of the areas in North-West London from which most of the patients in this study come. Many jobs are available, particularly for younger age groups. It is clear from the paper that many of the patients do work and it seems reasonable to say that at least in one sense the outcome in these patients has been better than that in the unemployed. Measures of outcome do not necessarily correlate. Perhaps the most disquieting finding of the study is the demonstration of better occupational outcome in patients with a short pre-treatment duration of illness who had been given placebo rather than active maintenance medication. In terms of relapse these patients did not do well but achieved their significantly better occupational outcome in the face of more relapses than similar patients prescribed active medication.

We hope that this letter provides the clarification that Dr Muijen seeks, but if there are any specific points he would like to discuss, he is most welcome to contact us.

University Department of Psychiatry

Eve C. JoHNSTONE

Royal Edinburgh Hospital

Edinburgh

Northwick Park Hospital

C. D. FRITH

Harrow

Middlesex

\section{Gender-identity clinic patients}

SIR: At a recent Journal Club meeting we discussed the paper by Burns et al (Journal, August 1990, 157, 265-268), which proved to be interesting but contained some surprising incongruities in the tables of figures used. We note the following unexplained discrepancies.

The "DSM-positive" column of Table 1, the whole of Table 2 and the whole of Table 3 refer to the same population (DSM-positive transsexuals included in this study; $n=77$ ). However, the breakdown of this group of people differed depending on which table you look at:

(a) In Tables 1 and 2 there are 20 members of the sample with a mean age of onset over 13,54 under 13, and three whose age of onset is "unknown". In Table 3 there are now 20 with mean age of onset over 13,53 under 13, and four "unknowns".

(b) In Tables 1 and 2 there are 27 members of the sample listed as heterosexual and 12 as "sexual orientation unknown". In Table 3, 26 are listed as heterosexual and 13 are "unknown".

(c) In Table 1 only one DSM-positive transsexual displayed sexual arousal with cross-dressing and 69 did not. In Table 2, seven DSM-positive transsexuals displayed sexual arousal with cross-dressing and 67 did not. In Table 3, 10 DSM-positive transsexuals displayed sexual arousal with cross-dressing and 63 did not. The remainder of the 77 people $(7,3$ and 4 respectively) were listed as "unknown" in each category.

(d) In Tables 1 and 3, 24 DSM-positive transsexuals were referred for surgery and 53 were not. In Table 2, 24 were referred for surgery, 49 were not and four were "unknown". 
We would be interested to hear the authors' response to these seeming inconsistencies.

N. E. DE TARANTo

A. Molivar

P. FLOWERS

J. SCOTT

E. ELSWORTH

M. JENKINS

M. Chapman

W. HOPKINS

D. TWENA

Department of Psychiatry

Chase Farm Hospital

Enfield, Middlesex

AUTHORS' REPLY: We are grateful to our colleagues at Chase Farm Hospital for pointing out the discrepancies in the Tables of our recent paper, and are flattered that the paper was chosen for such close scrutiny in what sounds like a stimulating Journal Club.

To answer the points:

(a) and (b) This was due to the erroneous transposition of one case (a heterosexual in the onset $<13$ group) from Tables 1 and 2 to the "unknown" category in Table 3.

(c) A proof error occurred in Table 2 and the column under core positive with regard to sexua arousal for cross-dressing should read 3, 30, 2 and not $0,33,2$. After this correction, Tables 2 and 3 then agree in that there were ten subjects with sexual arousal. The discrepancy between these tables and Table 1 is due to the definition used which was slightly different when assessing the DSM-III criteria and the presence of core transsexualism - in the former it was defined in terms of arousal with crossdressing (a narrower definition) whereby in the latter, a slightly wider definition was used to capture the sense of a total absence of sexual arousal in any aspect of the adoption of the cross-gender role.

(d) This was due to the addition of an "unknown" category to the "referral for surgery" in Table 2, whereas in Tables 1 and 3 these were included in the "not referred" group. To the best of our knowledge, these individuals were not referred for surgery.

While thanking Dr de Taranto and colleagues for bringing this to our attention, we feel these changes do not alter the main results or implications of our study.

Institute of Psychiatry

De Crespigny Park

London

\section{Musical and verbal hallucinations}

SIR: Berrios (Journal, February 1990, 156, 188-194) produced a useful literature review and statistical survey of cases of musical hallucinations. However, he does not seem to have read my related review of unilateral auditory hallucinations (Gordon, 1987) nor even my addendum in this journal (Gordon, 1988) drawing attention to it. Hence many interesting points were left unexplored and my conclusions were unchecked. Furthermore, it is now being cited as a 'definitive review' (Shapiro et al, 1991). To take some specific points:

Despite reporting personal cases with both musical and verbal hallucinations, Dr Berrios confined his review to musical ones only. Cases in the literature with otopathic auditory hallucinations cannot be clinically differentiated by type (i.e. music, words or elaborated tinnitus). Musical ones are admittedly quite striking given the specific and stirring quality of the evoked music. Indeed, it may not be coincidental that so many great composers had ear disease (Beethoven, Smetana, Schumann, etc). Nevertheless, most attention should be directed to non-musical auditory hallucinations, given their prominence in psychosis.

My references contained cases of musical hallucinations unused by $\mathrm{Dr}$ Berrios. There are undoubtedly others not cited by either of us.

I reported interesting relations between the laterality of the ear disease and hallucinations, but did not pursue this. Dr Berrios missed the chance to check this and did not even mention the subjective source of the hallucinations in any of his six new case vignettes.

My claim that hallucinations were more closely related to middle rather than inner ear deafness and to serous rather than purulent otitis was not assessed.

Dr Berrios made no distinction between unilateral and bilateral hallucinations. In medicine, causes of unilateral and bilateral diseases are often quite different. Focusing, as I did, on unilateral hallucinations simplifies correlation with ear or brain disease. Although I do not doubt that ear disease can produce bilateral hallucinations, it is very difficult to show this, even if true, from published reports.

He does not discuss the crucial question of whether ear disease without central disinhibition or neurological or psychiatric disease is a sufficient cause of auditory hallucinations. If so, concomitant brain disease may be a red herring. Dr Berrios claims a link between right hemisphere lesions and musical hallucinations, unsupported in recent well studied cases (Cambier et al, 1987; Shapiro et al, 1991). This makes it even more unfortunate that he had no comparable matching group of non-psychotic patients with verbal hallucinations linkable to left hemisphere lesions. 